Research Article

\title{
Application of Smartphones in Pavement Profile Estimation Using SDOF Model-Based Noisy Deconvolution
}

\author{
Amin Moghadam (iD and Rodrigo Sarlo \\ Department of Civil and Environmental Engineering, Virginia Tech, Blacksburg, VA 24061, USA \\ Correspondence should be addressed to Rodrigo Sarlo; sarlo@vt.edu
}

Received 16 October 2020; Revised 7 March 2021; Accepted 13 March 2021; Published 24 March 2021

Academic Editor: Hui Yao

Copyright (C) 2021 Amin Moghadam and Rodrigo Sarlo. This is an open access article distributed under the Creative Commons Attribution License, which permits unrestricted use, distribution, and reproduction in any medium, provided the original work is properly cited.

\begin{abstract}
The new generation of smartphones, equipped with various sensors, such as a three-axis accelerometer, has shown potential as an intelligent, low-cost monitoring platform over the past few years. This paper reports the results of an analytical and experimental study on a proposed SDOF model-based noisy deconvolution (SMND) coupled with a deechoing technique to estimate pavement profiles and to modify their geometry using a smartphone inside a vehicle. In the analytical study, the acceleration response of the car was obtained, where the input was a road profile with an arbitrary pattern. Two different methods, classical band-pass filter and wavelet-denoising technique, were used for denoising the acceleration response. In a 2-step deconvolution process coupled with a deechoing technique, the pavement profile was extracted and compared with the original pavement profile, demonstrating good agreement. In the next step, a parametric study was performed to evaluate the effect of vehicle characteristics and speeds. Then, a case study was conducted in Blacksburg, VA, to evaluate the capability of the proposed method in identifying profile types such as potholes and speed bumps. The acceleration-versus-time responses in vertical direction were recorded using smartphone accelerometers located in a moving vehicle. Then, the proposed approach was applied to remove the echo and vehicle dynamics effects to obtain the pavement profiles and to modify their geometry. The results showed that the proposed approach can remove the echo and vehicle dynamics effect from the response to obtain the pavement profile even if the vehicle characteristics and speed are changed.
\end{abstract}

\section{Introduction}

In general, pavement conditions affect the safety of drivers and passengers, ride comfort, handling stability, fuel consumption, vehicle maintenance costs, etc. The degraded road surface can cause traffic safety concerns and even pose accidents and loud noise emissions [1]. Road surface monitoring helps to maintain the road operations [2], and one way of road surface evaluation is pavement profile estimation. Pavement profile estimation can be used to compute the International Roughness Index (IRI) [3-6] to quantify road surface roughness or Pavement Serviceability Index (PSI) [7-9] to quantify the pavement quality and ride comfort. It can also be used to classify pavement deterioration types [10-12]. Laser profilers equipped with inertia sensors and cameras provide a high-accuracy, high-resolution option for pavement profile estimation $[13,14]$. However, because of their high operation costs, they cannot be used for regular road network monitoring. With the development of smartphone technology with built-in accelerometers, global positioning system (GPS), magnetometer, and gyroscopes, a new opportunity is provided for road surface integrity evaluation [15]. Compared to highresolution, costly devices, smartphones have shown potential as an intelligent, low-cost, crowd-based infrastructure alternative monitoring platform over the past few years. The smartphone's accessibility and affordability provide the crowdsourcing potentiality for data collection to cover the rapidly expanding road networks, and with more road users' participations, road integrity monitoring can become more efficient [15]. 
In general, existing methods for pavement profile estimation can be classified into four categories: noncontact measurement, direct measurement, neural-network-based estimation, and system dynamic response-based estimation [1]. The first two methods are more instrument-based, while the third and the last methods are computer-based and model-based, respectively. The noncontact measurement method uses lidar, laser profilers equipped with inertia sensors mounted on the vehicle to scan the pavement surface [16-19]. The advantage of this method is better transferability to regular vehicles that makes it suitable for real-time detection using regular vehicles. The drawbacks of the noncontact measurement method are their expensive installation and operational prices as well as their sensitivity to different weather conditions such that the results are not reliable in snowy and rainy weathers $[1,20,21]$.

The direct measurement method obtains the longitudinal profile of the road and measures other indicators such as International Roughness Index (IRI) along with the difference in elevation points using special vehicles equipped with road roughness meters such as longitudinal profile analyzer, profilometer, and so forth [22-25]. However, this method is usually used for road transportation maintenance and cannot be used for real-time measurements because of its inability to transfer to conventional vehicles. This restricts the number of potential users and data acquisition using smartphones such that it cannot be used to cover vast road networks.

The next class of methods is to use artificial neural network which is widely used in many different areas related to Intelligent Transportation Systems (ITS), Internet of Vehicles (IoV) [26], and traffic flow detection [27]. This is also used in pavement profile estimation and pavement deterioration classification [28-31], in which accelerometers are usually the key sensors used. It should be noted that although this method addresses some of the problems associated with the previous two methods (nontransferability and cost), there are some disadvantages associated with it as well. For instance, for machine training and network creation, a large number of datasets need to be collected in advance and over different roads. Also, besides a long computation process, this method needs many computing resources as well [1].

The last category is system dynamic response-based estimation [32-36]. In this method, besides the advantages of being low cost and transferable to regular vehicles, the problem associated with the neural network methods is also addressed such that there is no need to have a large number of data sets and a time-consuming computation process. A limited number of papers in this category (system dynamic response-based estimation) specifically propose pavement profile methods in which smartphones are used for data collection. In 2014, the acceleration data of a vehicle simulated as a quarter car was recorded using Android smartphones [6]. Then, the displacement response (vehicle dynamics and echo effects included) was obtained by double-integrating the acceleration response. The displacement response was input into the software program ProVAL for IRI estimation. In two similar studies conducted in 2019 and 2020, a smartphone measuring multiple outputs was used in which the vehicle was considered as a half-car model $[2,14]$. To identify the vehicle characteristics, in the first study [2], the response of the vehicle passing through a bump with known geometry was extracted, and genetic algorithm (GA) was then employed to determine the vehicle parameters. In the second study [14], the vehicle parameters were optimized by comparing the estimated profiles at the front and rear tires locations using GA. Then, augmented Kalman filter (AKF) was used for both studies for pavement profile estimation along with Rauch-Tung-Striebel smoothing (RTS) to improve the road profile estimation accuracy.

While the studies above demonstrate the potential of pavement estimation via a smartphone's dynamic response, however, some limitations still exist. System parameters such as passengers' mass, vehicle damping ratio, vehicle natural frequency, and so forth may change over time, causing the vehicle acceleration response variation with respect to time. Thus, the estimated pavement profile will be a function of the vehicle characteristics and the vehicle dynamics effects that should be properly removed. Also, in one of the studies discussed above [6], the vehicle was considered as a SDOF quarter-car model while a real vehicle has two sets of wheels, and as a result, when a vehicle moves through a speedbump, for instance, the estimated pavement profile will be a double speedbump (echo effect), one repeated with a delay after the first one. However, later, it will be shown that it is acceptable to consider the vehicle as a SDOF model to make the computation process simple, but some modifications will be needed. Also, while MDOF methods have demonstrated good performance in pavement profile estimation, they require extensive determination or calibration of various vehicle characteristics [14].

This paper presents a SDOF model-based noisy deconvolution (SMND) to provide a simple but robust pavement profile estimation approach which addresses the shortcomings discussed above. A key innovation of SMND approach is a two-step deconvolution process which removes vehicle dynamics effects from accelerometer measurements from a single smartphone inside a vehicle. The method avoids any pretests or training by extracting the deconvolution parameters (damping ratio and fundamental frequency) directly from operational vehicle vibration via Operational Modal Analysis (OMA) method. In this study, it was aimed to propose a simplified approach; thus, a SDOF quarter-car model was used to simplify the computational requirements. In order to deal with the limitations of this assumption introduced by two sets of wheels, another key feature of the proposed technique is to use a robust technique, "deechoing," to remove the undesirable effects while it still keeps the computation process simple.

In this paper, Section 2 introduces the OMA technique used to compute the vehicle characteristics, the SMND approach to remove the vehicle dynamic effects, and the deechoing technique to remove the echo from the estimated profile. Section 3 reports the results of a numerical study to show the significance of removing the vehicle dynamics effects and echo from the estimated pavement profile. Then, 
it is numerically shown that the proposed approach is capable of estimating the pavement profile, in a situation with and without noise, using the vehicle acceleration-time response passing through a pavement deterioration with an arbitrary pattern. Section 4 provides the results of a parametric study to evaluate the sensitivity of the results when vehicle characteristics and the vehicle speed change. Lastly, in Section 5, the results of the experimental study are presented to show the capability of the method in a real situation to obtain the pavement profile.

\section{Methodology}

In this section, the OMA technique, Covariance-Driven Stochastic Subspace Identification (SSI-Cov), is first introduced that was applied on the collected acceleration-time response of the vehicle to extract vehicle characteristics, i.e., damping ratio and fundamental frequency. Then, the methodology for pavement profile estimation is explained consisting of two steps: SDOF model-based noisy deconvolution (SMND) and deechoing. SMND was used to remove the vehicle dynamics effects from the displacement response. Deechoing was applied to the estimated pavement profile to remove the echo introduced by two sets of wheels.

\subsection{Covariance-Driven Stochastic Subspace Identification} (SSI-Cov). In the experimental study described later in Section 5, the vehicle characteristics (fundamental frequency and damping ratio) were obtained via SSI-Cov, which is a time-domain, Operational Modal Analysis (OMA) technique. This technique is described by Peeters and De Roeck [37], based on the original formulation by Van Overschee and De Moor [38]. A custom MATLAB code developed by Sarlo et al. [39] and available at a custom MATLAB library, https://code.vt.edu/vibes-lab/modal-analysis, was used to implement the technique.

In SSI-Cov technique, the user needs to choose the number of samples $(n)$, Hankel matrix block rows $(s)$, and a range of singular values $(r)$. In the experimental study, $s$ was chosen to be 20 and $n$ was 750 samples ( $7.5 \mathrm{~s})$. These values need to be large enough to average out the noise such that SSI-Cov produces visually observable results. Also, $r$ is twice the number of modes in the physical system.

The MATLAB code provides stabilization diagrams resulted from singular value decomposition (SVD) of the spectral density (SD) matrix. This is a plot of the number of modes $(m=r / 2)$ versus modal frequencies $(f)$. The tolerances used to define the stable/unstable natural frequencies and damping ratios were $\Delta f<0.07 \mathrm{~Hz}$ and $\Delta \zeta<0.11$. The results are provided in Section 5 .

2.2. SDOF Model-Based Noisy Deconvolution (SMND). This subsection explains how the proposed SMND approach removes the vehicle dynamics effects from the displacement-time response of the vehicle to obtain the pavement profile. Figure 1 depicts an example of the simulated vehicle model moving on pavement with an arbitrary profile pattern.

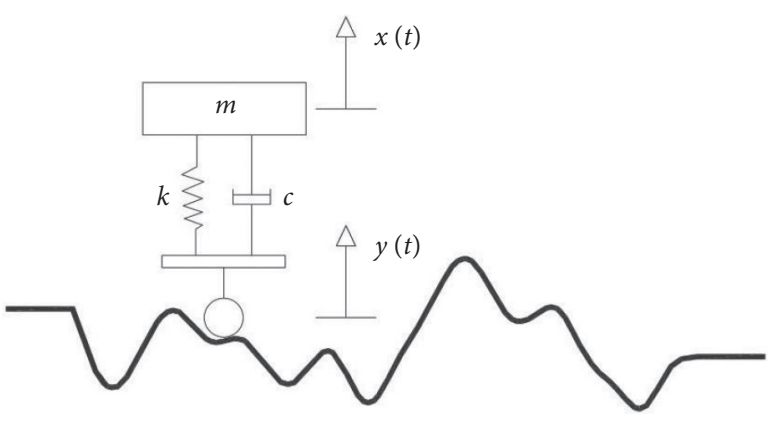

FIgURE 1: SDOF model of the vehicle.

Because the acceleration recorded by the accelerometers is the absolute acceleration response, the equation of motion of the vehicle is written as

$$
m \ddot{x}+c(\dot{x}-\dot{y})+k(x-y)=0,
$$

in terms of absolute displacement $x$ and ground displacement $y$. Equation (1) can be manipulated to be written in the simplified form

$$
m \ddot{x}+c \dot{x}+k x=f(t)=k y+c \dot{y},
$$

where $y$ and $\dot{y}$ are the pavement height and the pavement height change with respect to the time, respectively.

Also, considering the impulse response of the vehicle, equation (2) can be written in a convolution form,

$$
x(t) * h_{1}(t)=f(t)=k y+c \dot{y},
$$

where

$$
h_{1}(t)=\frac{1}{m \omega_{d}} e^{-\zeta \omega_{n} t} \sin \omega_{d} t
$$

is the impulse response function. This is only a function of the vehicle dynamic characteristics. Additionally, $\varsigma, \omega_{n}$, and $\omega_{d}$ in equation (4) are the vehicle damping ratio, natural frequency, and the damped natural frequency, respectively. Knowing that the initial vertical displacement and velocity of the vehicle before going through the pavement deterioration are zero, the Laplace transform of the force equation, $f(t)=$ $k y+c \dot{y}$, will be

$$
k Y(s)+c s Y(s)=F(s),
$$

where $Y(s)$ is the Laplace transform of $y(t)$ and $F(s)$ is the Laplace transform of $f(t)$ computed in the previous step. Then, from equation (5), $Y(s)$ can be calculated as

$$
Y(s)=H_{2}(s) \cdot F(s),
$$

where $H_{2}(s)=1 /(c s+k)$ is the Laplace transform of the impulse function, $h_{2}(t)$. Taking the inverse Laplace transform of $\mathrm{H}_{2}(s), h_{2}(t)$ will be obtained; that is,

$$
h_{2}(t)=\frac{1}{c} e^{-k t / c} .
$$

In the final step, from equation (6), $y(t)$ can be extracted using the convolution property, saying that the convolution of two functions in the time-domain is equal to the 
multiplication of them in the frequency-domain and vice versa. Thus,

$$
y(t)=f(t) * h_{2}(t)
$$

The absolute displacement $x(t)$ can be computed by double integration of the denoised acceleration-time response recorded by the smartphone accelerometer. Then, the force applied to the vehicle, $f(t)=k y+c \dot{y}$, can be computed by convolving the absolute displacement, $x(t)$, with the impulse response function $h_{1}(t)$ defined in equation (4). Once the force $f(t)$ is computed, the ground displacement, $y(t)$, can be obtained by convolving the force with $h_{2}(t)$ defined in equation (7). The term $y(t)$, once corrected for vehicle velocity, represents the pavement profile with vehicle dynamics effects removed. Figure 2 provides a diagram summarizing the proposed SMND approach.

2.3. Deechoing Technique. In the proposed method, the vehicle was considered as a SDOF model (quarter-car model) to make the computation process simple. The proposed deechoing technique solves some of the limitations of this model but still keeps the computation process simple. A real vehicle has two sets of wheels, front row and rear one. Hence, when a vehicle moves through a speedbump, for instance, the estimated pavement profile will be a double speedbump, with spacing corresponding to the axle spacing of the vehicle. In the time-dependent version of the pavement profile (before correcting for speed), this spacing may be treated as a delay or an "echo." One of the applications of convolution is removing the echo (repetition with a delay) from a signal by deconvolving it with an echo impulse function; a sequence of numbers is defined as

$$
h_{3}(t)=\left[1, M, \text { zeros, } \frac{\mathrm{Peak}_{2}}{\mathrm{Peak}_{1}},\right]
$$

where $M$ is the delay (the number of samples) between the starting point of the main signal and the echo in the pavement profile in which dynamics effects are removed. Peak $_{1}$ and Peak $_{2}$ are the absolute positive or negative peak values of the main signal and the echo, respectively. This accounts for any differences in amplitude in the response between the front and rear wheels. To this end, the response segment with echo should be manually selected and deconvolved with the impulse function to remove the echo effect. Then, the rest of the response (before and after the selected response segment) will be attached to the deechoed segment with zeros substituted for the removed subsegment.

\section{Numerical Study}

3.1. No Noise Added. In the first step, the approach was numerically simulated to assess the validity of the approach. All the computation process was done in MATLAB [40]. This first simulation ignores the effects of noise. This is done to show that the proposed method concept works properly. Before going through more complex pavement profiles, it is important to illustrate how the vehicle dynamics included in the response can distort the estimated pavement profile using a simple example with the pavement geometry shown in Figure 3(a). Also, it should be noted that the acceleration-time response of the vehicle moving through a double speedbump (Figure 3(b)) is obtained to simulate the effect of two sets of vehicle wheels (front and rear). The Newmark linear acceleration method [41] was used to obtain the vehicle responses using a code developed by Moghadam et al. [42]. Figure 3(c) illustrates the result of double integration of the acceleration-time response of a vehicle with the fundamental frequency of $1.18 \mathrm{~Hz}$ and damping ratio of 0.5 and moving with a constant speed of $2.24 \mathrm{~m} / \mathrm{s}$. Other speeds are discussed in Section 4.2. In this response, the vehicle dynamics effects as well as echo are still included. According to Figure 3(c), the displacement response contains two speedbumps (echo effect) and negative displacements right after them caused by vehicle dynamics effects. Furthermore, the heights of the speedbumps were also overestimated, which is another undesirable effect of the vehicle dynamics. However, applying SMND and deechoing, Figure 3(d) was resulted, where the negative displacement and the echo have completely disappeared, and the geometry of the speedbump was also modified. As a result, it is beneficial to remove the echo and vehicle dynamics effects from the displacement response obtained by double integration before using it for any purpose, including pavement deterioration classification, IRI computation, and PSI computation.

In the next step, it is required to numerically evaluate the proposed method on a more complex example to see if it is capable of estimating the pavement profile with any arbitrary pattern. Echo effect is ignored in this example. Figure 4 shows that the estimated pavement profile matches very closely with the exact profile. It should be noted that the negligible difference which is less than $0.3 \mathrm{~cm}$ between the estimated pavement profile and the exact pavement profile shown in Figure 4 is because of the Newmark method approximation.

3.2. Noise Added. So far, it is shown that the proposed method works well when there is no noise in the acceleration-time measurement. However, there is no doubt that noise is always involved in the measurement, and it usually influences the results' accuracy. The purpose of this section is to show that the proposed SMND approach works properly to estimate the pavement profile even for a high-noise case. Thus, as shown in Figure 5, the acceleration-time response using Newmark method was contaminated with a considerable level of white noise using random numbers generated in MATLAB.

In this study, two noise removal techniques were used to be coupled with the proposed SMND approach for pavement profile estimation, and the results were compared: wavelet-denoising technique and band-pass filter. For wavelet-denoising technique, a function in MATLAB, "wnoise," was used. For noise removal using a band-pass filter, Fast Fourier Transform (FFT) of the noisy acceleration-time response was taken to distinguish between 


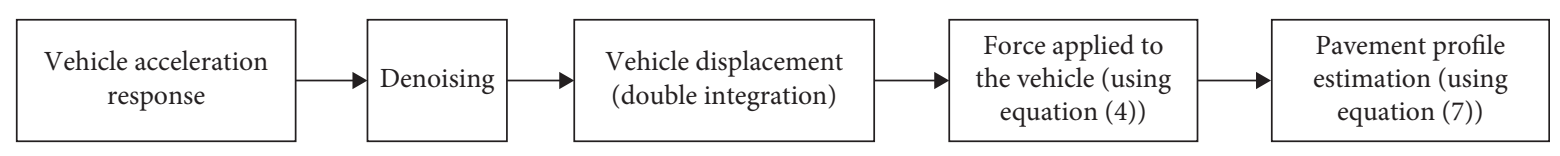

FIGURE 2: Flowchart of the proposed SMND approach for pavement profile estimation.

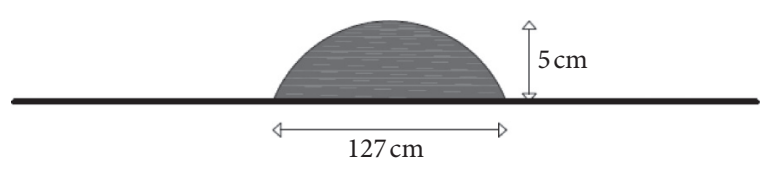

(a)

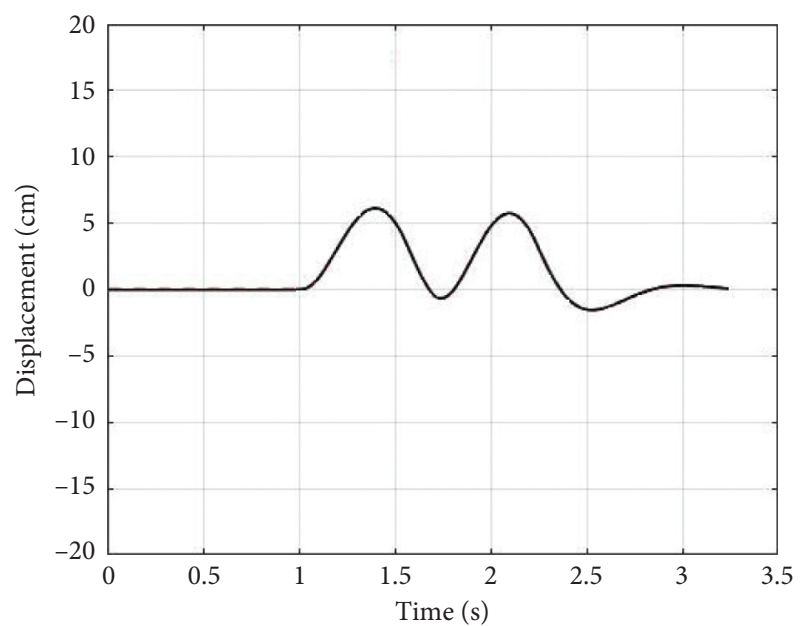

- - - Displacement (double integration)

— Original displacement (Newmark)

(c)

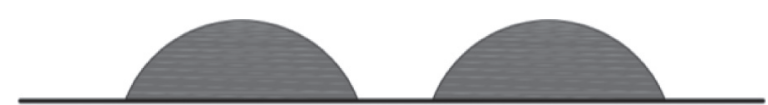

(b)

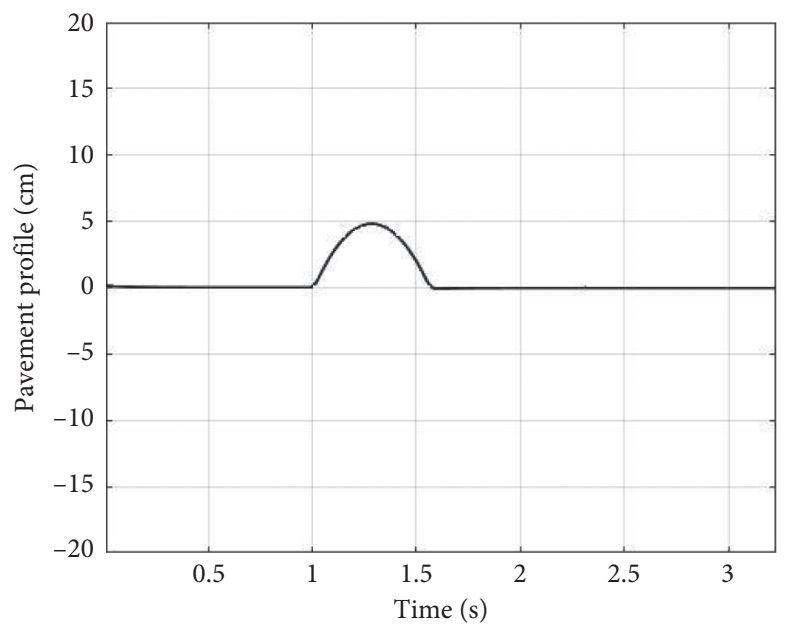

(d)

FIgURe 3: Numerical pavement profile estimation (no noise included). (a) The speedbump geometry. (b) The input pavement profile. (c) Displacement response (echo and vehicle dynamics effects included). (d) Pavement profile estimation (deechoed and vehicle dynamics removed).

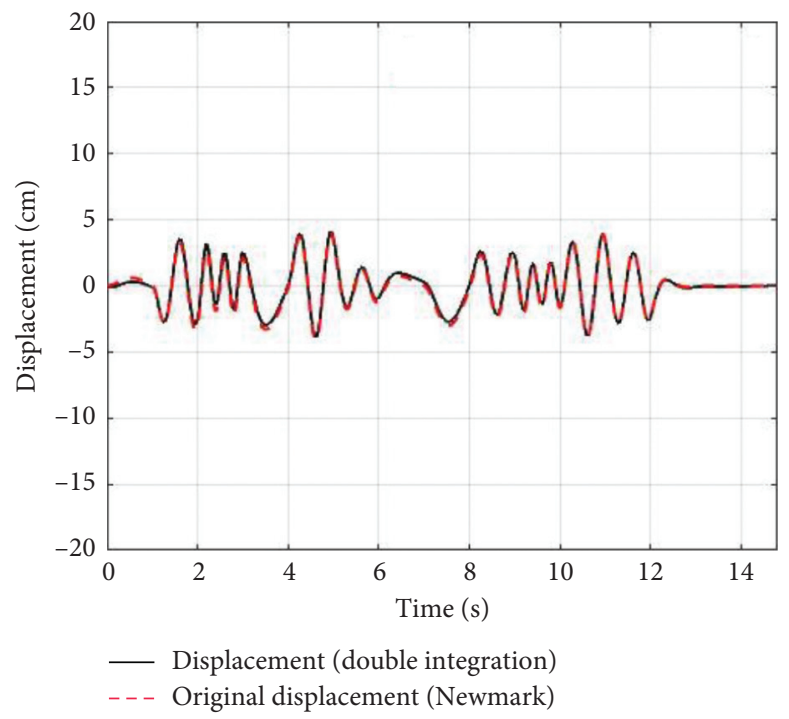

FIgURE 4: Pavement profile estimation using the proposed SMND approach (no noise included). 


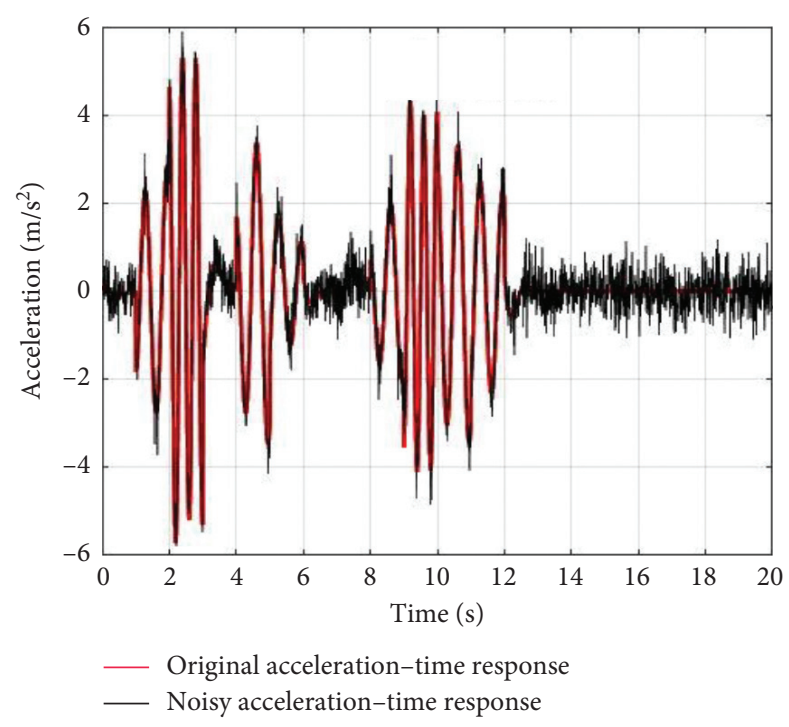

FIGURE 5: Noisy acceleration-time response.

the frequency range corresponded to the vehicle response and the frequency range of the noise. The frequency range associated with the vehicle response (1 to $3.3 \mathrm{~Hz}$ ) was extracted using a band-pass filter. Once the denoised acceleration-time responses were obtained, double integration method was then employed to find the vehicle displacement. To remove the inconvenient effects of the remaining noise in the acceleration-time response obtained using wavelet-denoising technique, a high-order polynomial curve (order of 14 in this study) was fitted to the displacement response. Then, in the last step, the proposed SMND approach was applied to the denoised displacement response to remove the vehicle dynamics effects and to find the pavement profile.

Figures 6(a) and 6(b) compare the exact pavement profile and the estimated ones using wavelet-denoising technique and band-pass filter, respectively. Although there was a considerable level of noise in the acceleration-time response, the proposed SMND approach still works well for pavement profile estimation. According to Figures 6(a) and 6(b), the maximum errors in pavement profile height estimation were approximately 1.3 and $2.3 \mathrm{~cm}$ for waveletdenoising technique and band-pass filter, respectively, which can be considered acceptable for the matter of pavement profile estimation. A greater error for the band-pass filter occurred because the cutoff band was selected manually to reduce the overall estimation error, and this involved cutting off some of the vehicle dynamics. This can be considered a drawback for the band-pass technique. In fact, for using band-pass filter, for each single recorded response, one needs to put a lot of effort to manually remove the frequencies associated with the noise through a trial-and-error process to ensure that the vital frequencies corresponded to the vehicle response are not removed. However, unlike the band-pass technique, using wavelet-denoising technique, a MATLAB code can be implemented to automatically remove the noise from many recorded responses. Thus, for the rest of this study, wavelet-denoising technique is used.

\section{Parametric Study}

So far, it is demonstrated that the proposed SMND approach works properly. However, there are other factors that may affect the results, such as the vehicle characteristics and speed. These are addressed here.

4.1. Vehicle Characteristics Effect. To consider the vehicle characteristics effects, twenty different simulated vehicles with different characteristics were considered. In the first ten cases, damping ratios were constant $(\zeta=0.5)$ while the fundamental frequencies were varied between 7.32 to $10.67 \mathrm{rad} / \mathrm{s}$ to consider the effect of fundamental frequency on the pavement profile estimation results. In the second ten cases, fundamental frequencies were constant $\left(\omega_{n}=9.68 \mathrm{rad} /\right.$ s) and the damping ratios were changed between 0.3 and 0.65 to consider the effect of damping ratios on the results. For each case, the pavement profile was estimated using the acceleration-time response of the vehicle passing through the pavement profile shown in Figure 4. No noise was added to the responses to only consider the vehicle characteristics effects on the pavement profile estimation. Figures 7(a) and 7(b) show the mean-squared-error (MSE) of the estimated pavement profiles with respect to the exact pavement profile for different values of fundamental frequencies and damping ratios, respectively. According to Figure 7(a), the greater the fundamental frequency is, the greater MSE is, while Figure 7 (b) shows that increasing the damping ratio reduces the error.

To visually observe how the errors may distort the results, the estimated pavement profiles for $\omega_{n}=10.67 \mathrm{rad} / \mathrm{s}$ as well as $\zeta=0.3$, which are the worst cases with maximum MSEs, are plotted in Figures 8(a) and 8(b), respectively. According to Figures 8(a) and 8(b), both cases are in great agreement with the exact pavement profile. The maximum error for $\omega_{n}=10.67 \mathrm{rad} / \mathrm{s}$ was about $0.5 \mathrm{~cm}$ while it was about $0.8 \mathrm{~cm}$ for $\zeta=0.3$, where the maximum error is defined as the 


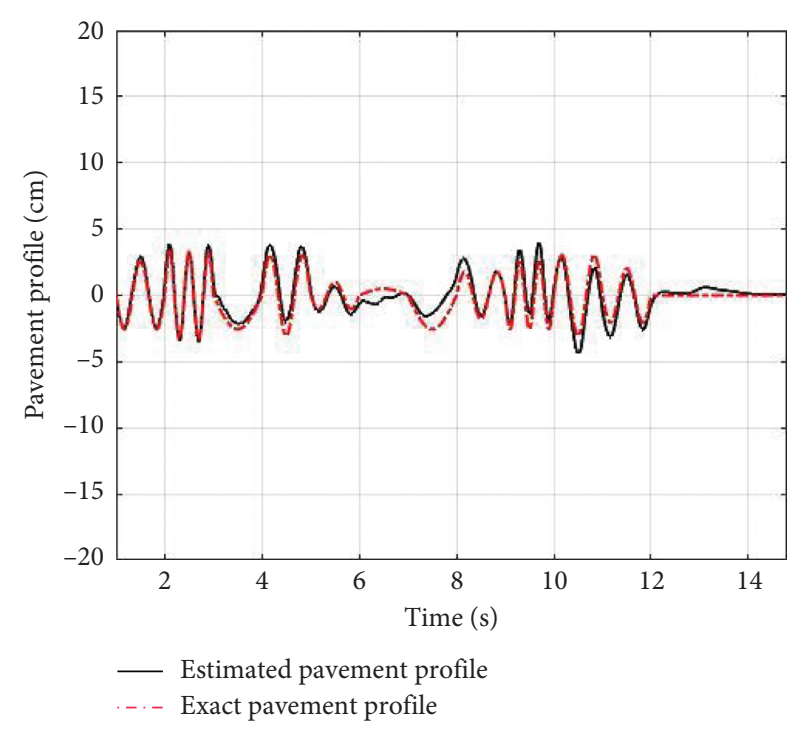

(a)

FIGURE 6: Pavement profile estimation (noisy response).

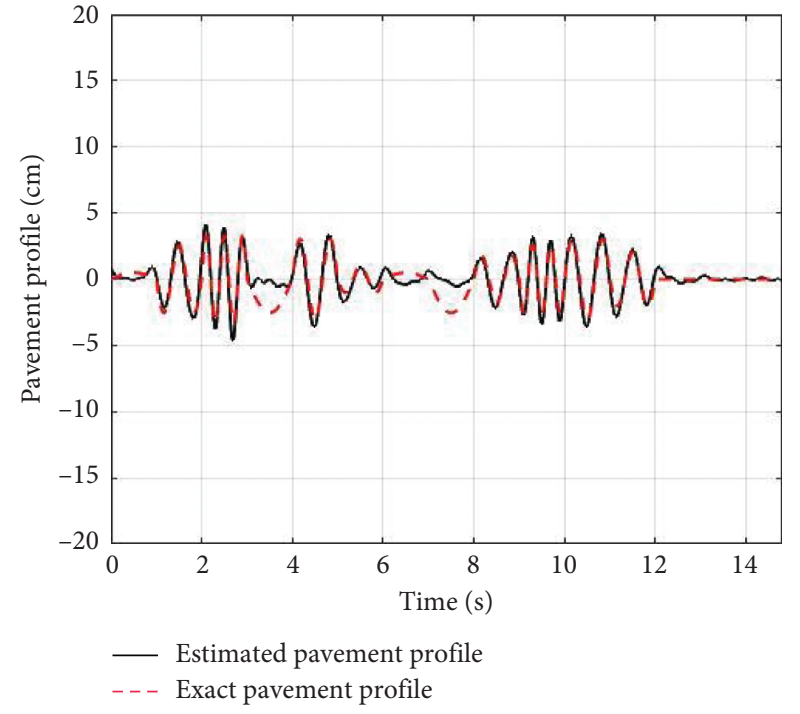

(b)

(a) Wavelet-denoising technique. (b) Band-pass filter.

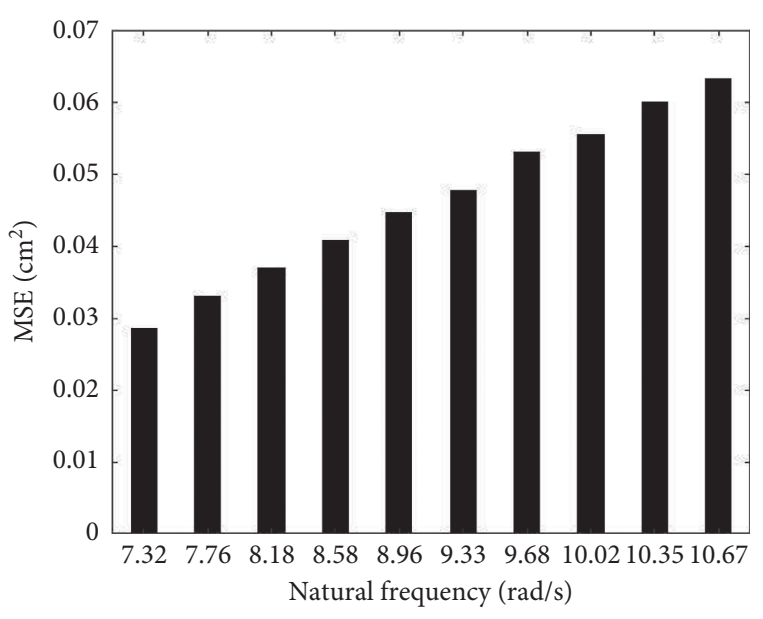

(a)

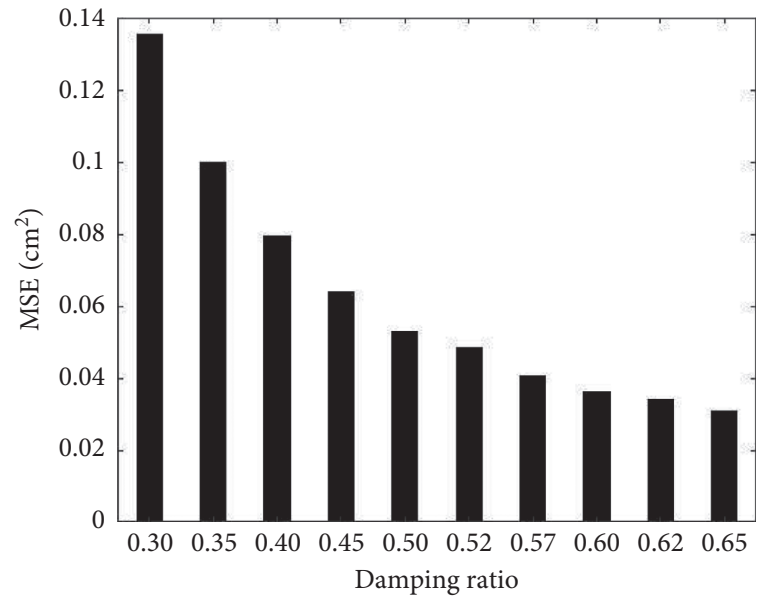

(b)

FIGURE 7: The pavement profile error for different values of fundamental frequency and damping ratio. (a) Fundamental frequency variation $(\zeta=0.5)$. (b) Damping ratio variation $\left(\omega_{n}=9.68 \mathrm{rad} / \mathrm{s}\right)$.

maximum distance between the estimated pavement profile with the exact one. So, it can be claimed that even if one uses a different vehicle with different characteristics, the proposed method will still be capable of vehicle dynamics effects removal and pavement profile estimation. This claim is assuming low noise, but even in a noisy condition, it was already shown that the maximum error is $1.3 \mathrm{~cm}$ when wavelet denoising is used.

4.2. Vehicle Speed Effect. The next factor that needs to be considered is vehicle speed. There are many ways to numerically simulate the vehicle speed variation on the pavement profile estimation. To numerically increase and decrease the vehicle speed, the input pavement profile shown in Figure 4 (amplitudes multiplied by 2 ) with a total length of
$10.2 \mathrm{~m}$ was compressed and stretched, respectively. The vehicle speeds considered in this study were 2.6, 5.1, 6.0, 7.8, $9.4,11.1$, and $13.6 \mathrm{~m} / \mathrm{s}$. No noise was added to the responses for a better observation of the vehicle speeds' effects on the results. Figure 9 shows the mean-squared-error (MSE) of the estimated pavement profiles with respect to the exact pavement profile for different vehicle speeds. According to Figure 9, the higher the vehicle speed, the greater MSE.

To visually observe how different speeds may distort the results, the estimated pavement profiles for four different speeds are demonstrated in Figures 10(a) through 10(d). According to these figures, the speed is a relatively effective factor such that the maximum error is $5.5 \mathrm{~cm}$ for the speed of $13.6 \mathrm{~m} / \mathrm{s}$, where the maximum error is defined as the maximum distance between the estimated pavement profile with the exact one. However, according to Figures 10(a) 


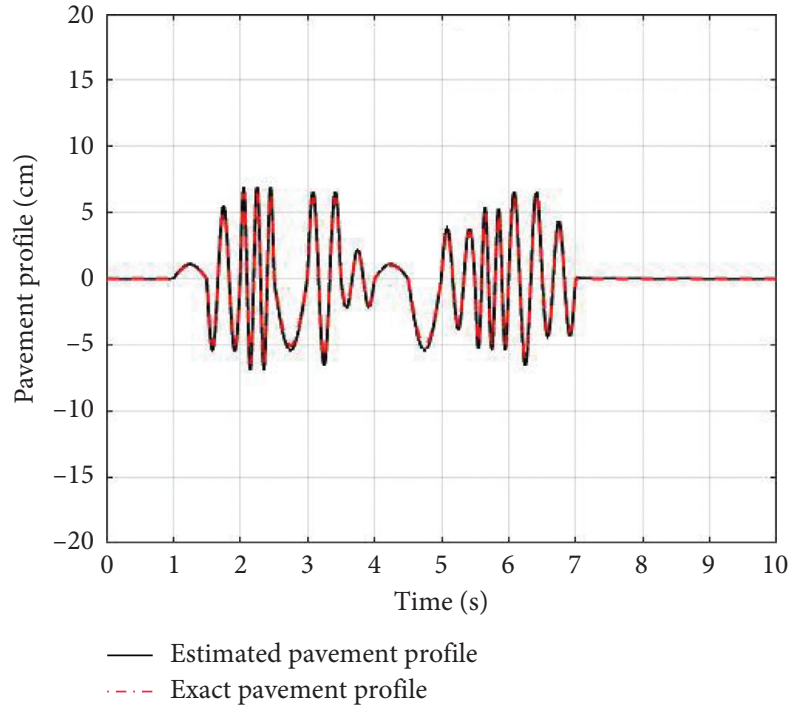

(a)

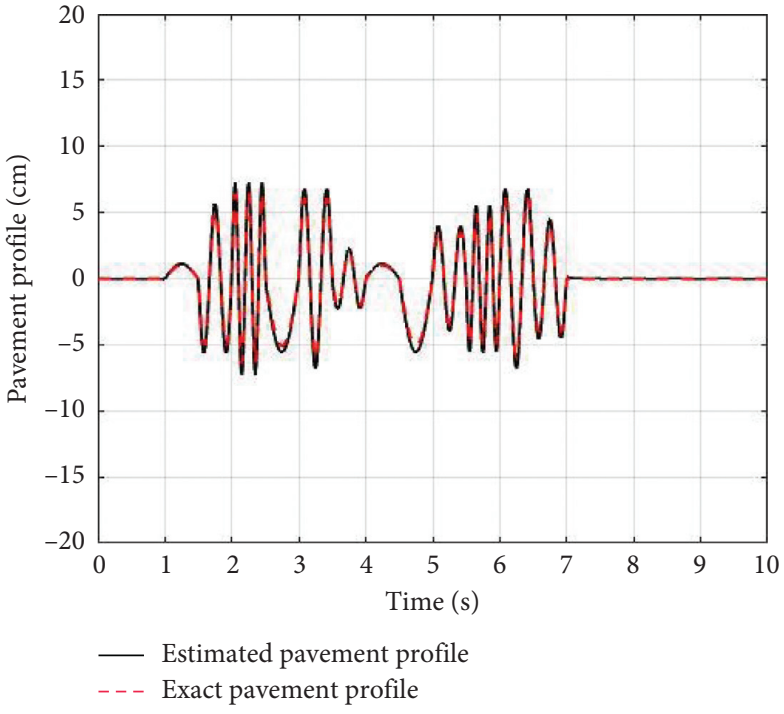

(b)

FIgURE 8: The estimated pavement profile for two cases with maximum MSEs shown in Figure 7. (a) $\omega_{n}=10.67 \mathrm{rad} / \mathrm{s}$ and $\zeta=0.5$. (b) $\omega_{n}=9.68 \mathrm{rad} / \mathrm{s}$ and $\zeta=0.3$.

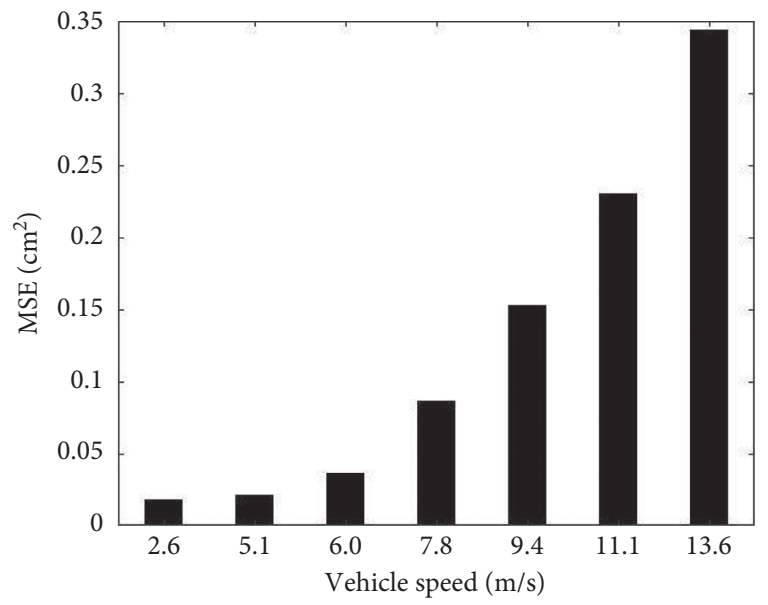

FIGURE 9: The pavement profile estimation error for different speed values.

through $10(\mathrm{~d})$, the estimated pavement profiles are in good agreement for the speeds of less than $7.8 \mathrm{~m} / \mathrm{s}$ with a maximum error of $2.4 \mathrm{~cm}$ and more sensitive to speeds greater than $7.8 \mathrm{~m} / \mathrm{s}$ with a maximum error of $5.5 \mathrm{~cm}$. Yet, no one chooses a vehicle speed of $7.8 \mathrm{~m} / \mathrm{s}$ or more to drive through the unusual pavement profile specified in Figure 4. In fact, the higher speeds were only chosen for a research purpose. As a result, although the vehicle speed can be influential on the results, for reasonable operational speed which is less than $7.8 \mathrm{~m} / \mathrm{s}$ in this pavement profile case, the results are reliable. The estimated pavement profile becomes more distorted at high vehicle speeds (greater than $7.8 \mathrm{~m} / \mathrm{s}$ ) due to the high frequency of the pavement profile compared to the vehicle's natural frequency. Thus, the pavement displacement is absorbed primarily in the suspension spring and very little is transferred to the vehicle body and the accelerometer. As observed in Figure 10(d), this results in an underestimated pavement profile, particularly for highfrequency displacements.

\section{Experimental Study}

In order to test the methodology in a real situation, an experimental study was conducted in Blacksburg, VA. A smartphone, iPhone XR, was fixed on the vehicle armrest box (between the two front seats) in a Hyundai Elantra 2011. First, SSI-Cov was used to determine the vehicle characteristics. Figure 11 shows the stabilization diagram resulted from the MATLAB code described in Section 2.1. According to Figure 11, only one stable mode was detected with a fundamental frequency of $1.5 \mathrm{~Hz}$ and damping ratio of about 0.33 .

Two different pavement types, such as a speedbump and a pothole, were considered. Figure 12 shows the pictures of the speedbump and pothole used in this study. The 


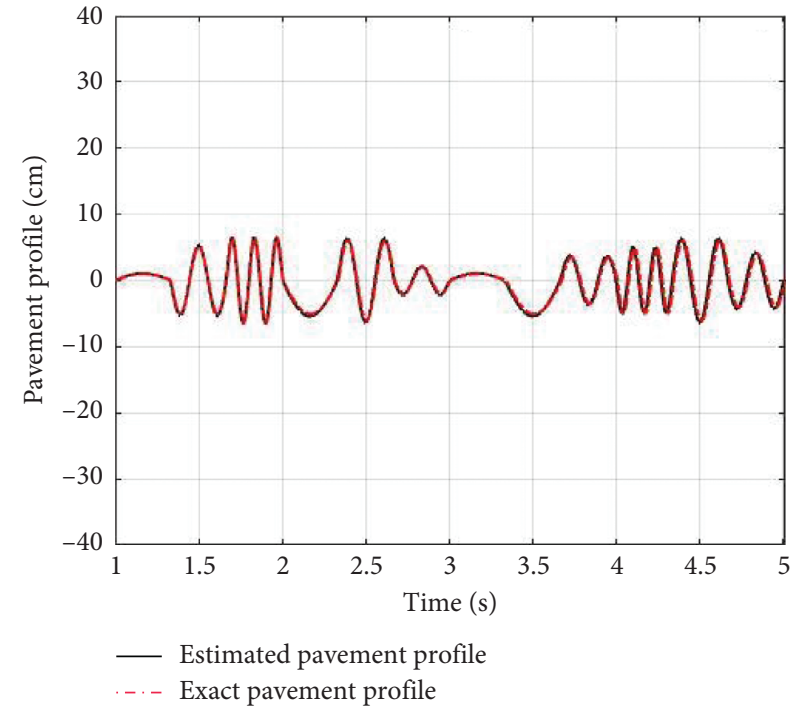

(a)

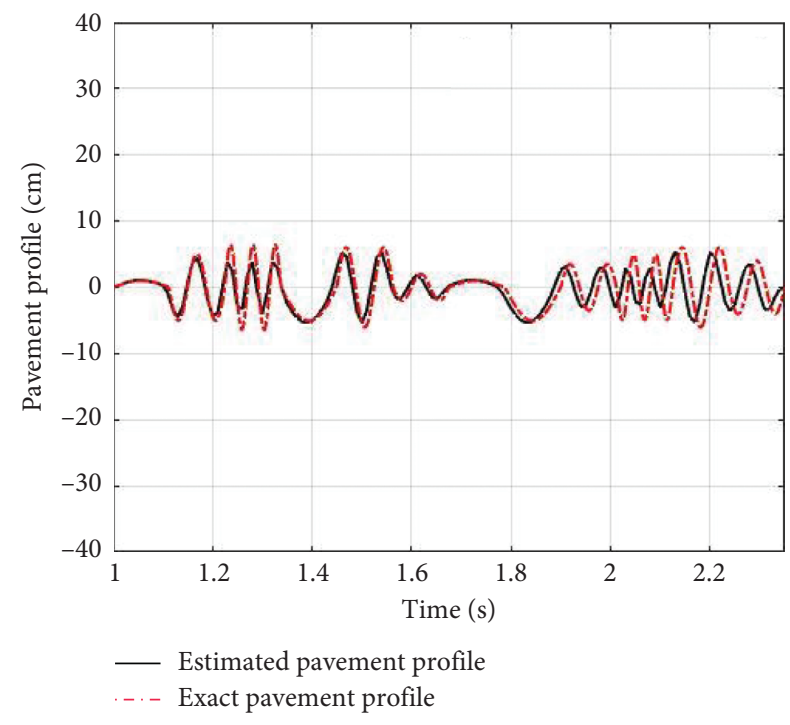

(c)



(b)

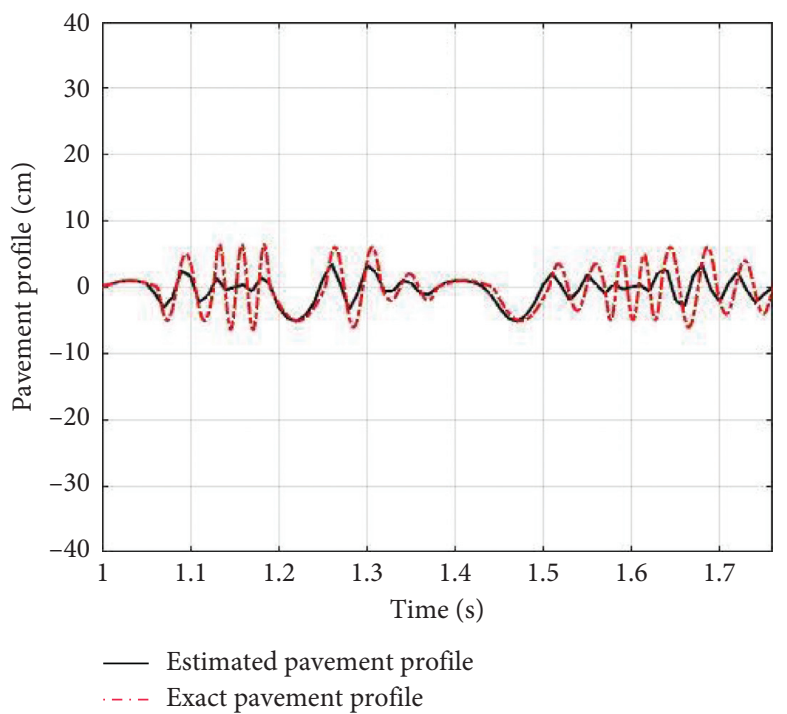

(d)

Figure 10: The estimated pavement profile for four different vehicle speeds. (a) Vehicle speed of $2.6 \mathrm{~m} / \mathrm{s}$. (b) Vehicle speed of $5.1 \mathrm{~m} / \mathrm{s}$. (c) Vehicle speed of $7.8 \mathrm{~m} / \mathrm{s}$. (d) Vehicle speed of $13.6 \mathrm{~m} / \mathrm{s}$.

acceleration-time responses of different cases in the vertical direction were recorded with a sampling rate of 100 samples per second, which is the maximum possible sampling rate of iPhone XR. The vehicle speed for all cases was about $2.24 \mathrm{~m} / \mathrm{s}$ because it was not practical to run the vehicle with higher speeds over a speedbump or a pothole. Higher speeds were already considered in the numerical study discussed in Section 4.2. In this study, smartphone version of MATLAB was used to store the responses in the cloud. Then, after the noise of each response was removed using the waveletdenoising technique, the proposed SMND approach was applied to remove the vehicle dynamics effects, to modify the pavement geometry, and to estimate the pavement profile.

Furthermore, the speedbump has a height and a horizontal length of, in average, 7.5 and $104 \mathrm{~cm}$, respectively, determined by site measurement. Figure 13(a) (black profile) shows the estimated pavement profile with echo and vehicle dynamics effects removed versus the displacement response when echo and vehicle dynamics effects are still included (red profile). This is to show how vehicle dynamics effects can distort the estimated pavement profile. According to Figure 13(a), when the echo and vehicle dynamics effects are removed, the estimated geometry shown in Figure 13(b) has been closer to the real geometry with a maximum error of about $2.2 \mathrm{~cm}$ and $9 \mathrm{~cm}$ in height and horizontal length estimations, respectively. This shows that the proposed SMND method coupled with the deechoing technique is capable of removing echo and vehicle dynamics effects and modifying the geometry and finally estimating the pavement profile even in a real situation.

The proposed method was then applied to the pothole as well. Based on site measurement, the height and horizontal 


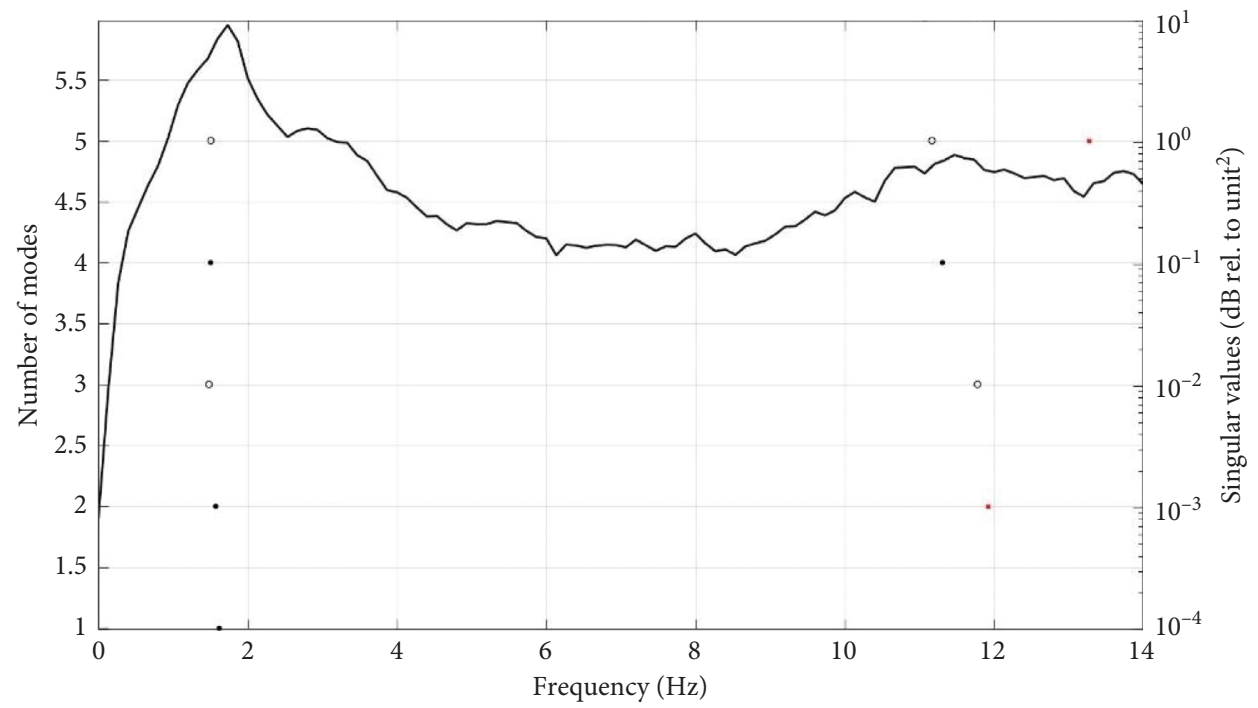

- Stable

- Damping unstable

- Unstable

FIgure 11: The stabilization diagram for Hyundai Elantra 2011.

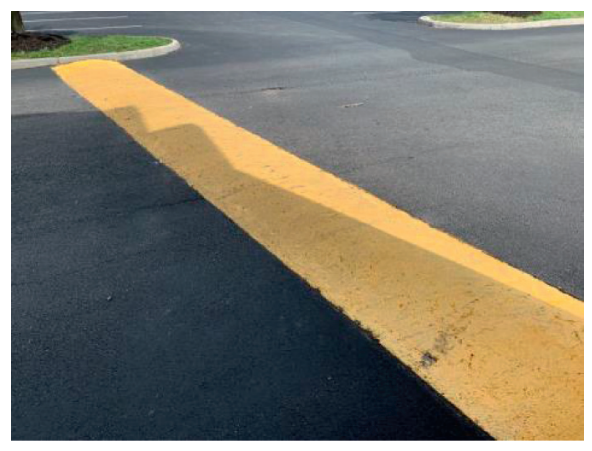

(a)

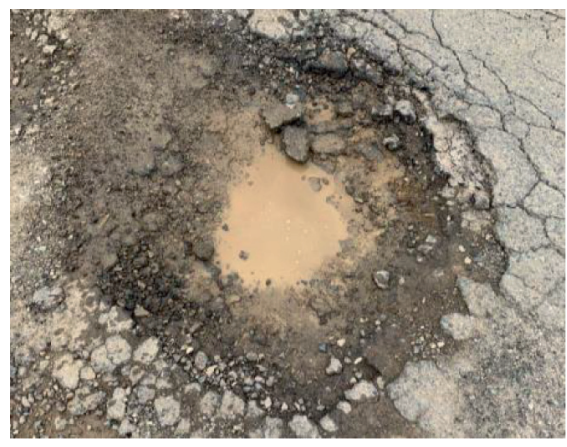

(b)

FIgURE 12: Pavement deterioration samples in Blacksburg, VA. (a) Speedbump sample. (b) Pothole sample.

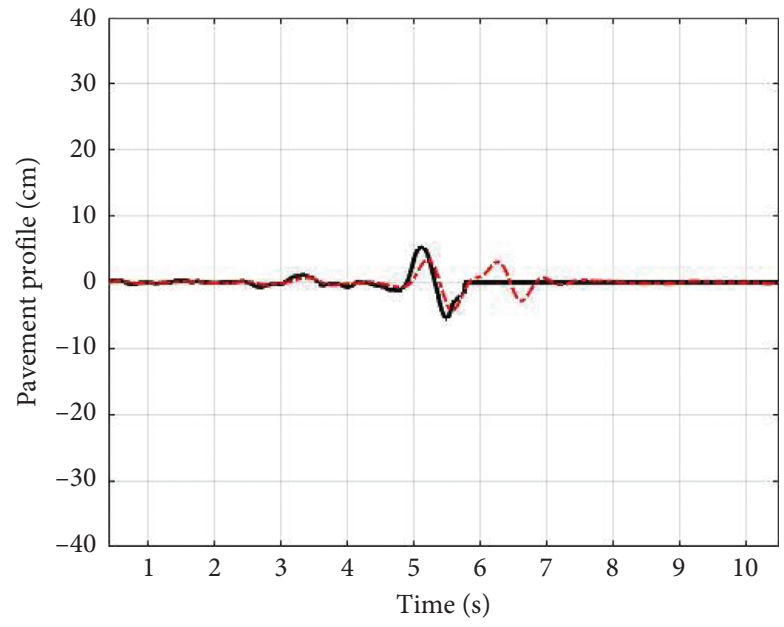

Estimated pavement profile Double integration

(a)

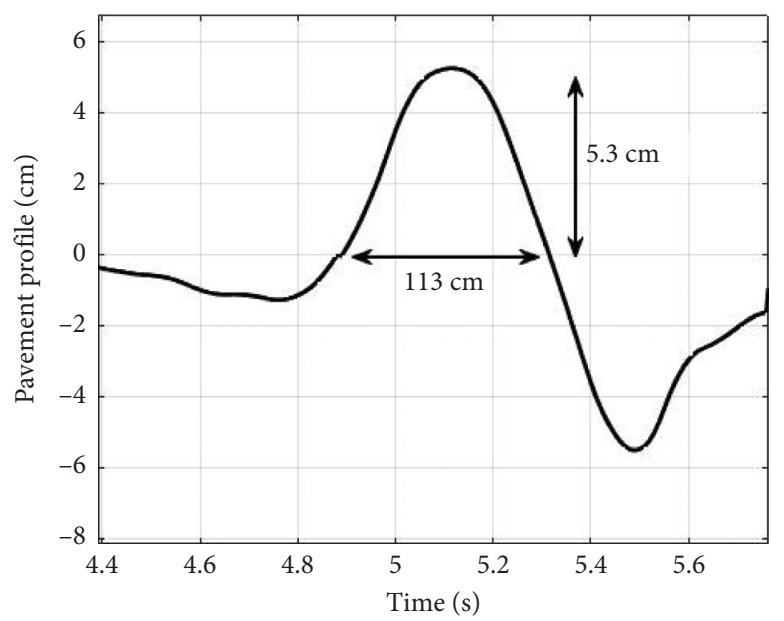

(b)

Figure 13: Pavement profile estimation for the speedbump. (a) The estimated pavement profile versus double integration (speedbump). (b) The estimated geometry (speedbump). 


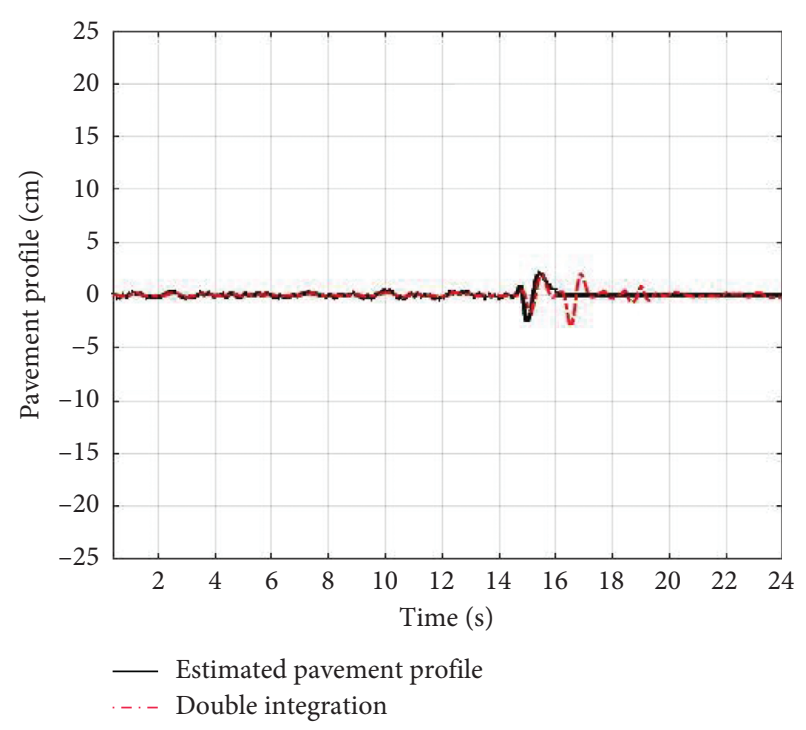

(a)

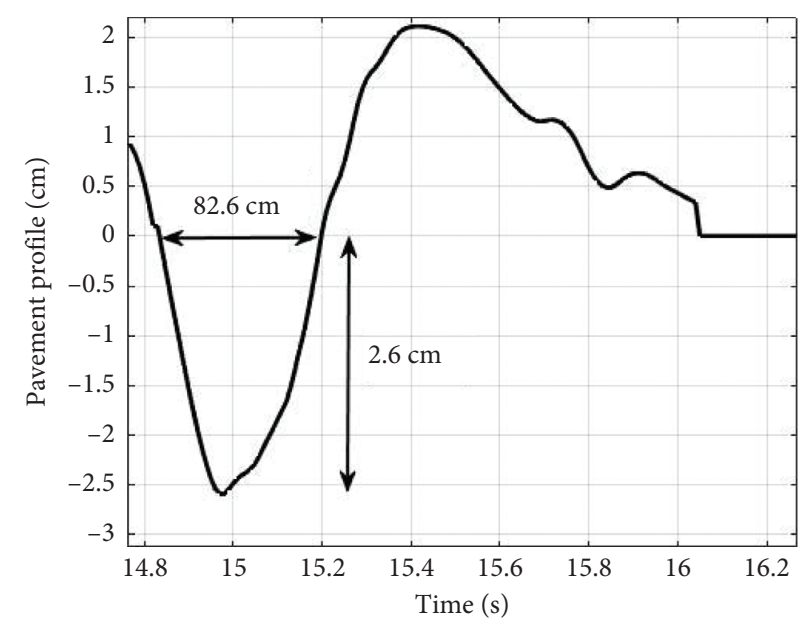

(b)

Figure 14: Pavement profile estimation for the pothole. (a) The estimated pavement profile versus double integration (pothole). (b) The estimated geometry (pothole).

length of the pothole sample were in average about $4 \mathrm{~cm}$ and $94 \mathrm{~cm}$, respectively. Figures 14(a) and 14(b) show the estimated pavement profile with echo and vehicle dynamics effects removed (black profile) versus the displacement response when echo and vehicle dynamics effects are still included (red profile). According to Figure 14(b), the estimated pavement profile is in good agreement with the exact profile with a maximum error of about 1.4 and $11.4 \mathrm{~cm}$ in height and horizontal length estimations, respectively. This shows that removing the echo and vehicle dynamics effects from the displacement response will modify the geometry of the estimated pavement profile. It should be noted that one issue in the pavement profile estimation shown in Figure 14(a) is the positive displacement right after the pothole caused by the pitching mode of the vehicle. This is not accounted for by the SDOF model, but could be removed by considering the vehicle as a half-car model in future studies.

Also, it should be noted that the reason why the results are only compared with double integration is because it was aimed still to take advantage of simple methods such as double integration, but it was also aimed to solve their current problems such as echo and dynamic effects. Other complex methods in the literature are ignored because they were against the simplicity purpose.

\section{Conclusions and Future Study}

In this study, SDOF model-based noisy deconvolution (SMND) approach coupled with a deechoing technique was proposed to remove vehicle dynamics effects for pavement profile estimation and to modify the estimated geometry. According to the numerical study, the SMND approach was capable of removing the vehicle dynamics effects from response recorded via smartphone accelerometers even in a pretty noisy environment in both numerical and experimental cases. The SMND approach was also able to specify if there was a pothole or speedbump on the pavement and to estimate its geometry properly. Furthermore, when there was a pothole/speedbump on the pavement, as a result of having two sets of wheels, two potholes/speedbumps appeared in the estimated profile (called "echo" in this study). However, the deechoing technique properly removed the echo effects from response and still kept the computation process simple so that only a single pothole/ speedbump appeared in the final estimation. According to the parametric study, vehicle characteristics, i.e., damping ratio, weight, and suspension system stiffness, did not significantly impact the estimated pavement profile, and using the proposed SMND approach, vehicle dynamics effects removal and pavement profile estimation were successfully done regardless of the vehicle type. On the other hand, the vehicle speed could significantly affect the pavement estimation results, with a maximum error of $5.5 \mathrm{~cm}$ for the speed of $13.6 \mathrm{~m} / \mathrm{s}$. However, within reasonable vehicle operational speeds to move through a speedbump and a pothole which is less than $7.8 \mathrm{~m} / \mathrm{s}$ in this study, the results were reliable with a maximum estimation error of about $2.4 \mathrm{~cm}$.

In this paper, as a proof-of-concept study, the examples considered in the experimental section were intentionally chosen to be simple because complex pavement profiles, due to their shape complexity, are not able to show how dynamics effects and echo can change the estimated profile and why echo and dynamic effects removal is so important. However, as a future experimental study, more complex pavement profiles should also be considered to evaluate the proposed method further.

\section{Data Availability}

The custom MATLAB code used for obtaining modal parameters, i.e., damping ratios and frequencies, is available at 
a custom MATLAB library, https://code.vt.edu/vibes-lab/ modal-analysis.

\section{Disclosure}

The sponsor did not have any involvement in the study design; in the collection, analysis, and interpretation of data; in the writing of the report; or in the decision to submit the article for publication.

\section{Conflicts of Interest}

The authors declare that they have no known competing financial interests or personal relationships that could have appeared to influence the work reported in this paper. Submitting authors are responsible for coauthors declaring their interests.

\section{Acknowledgments}

The authors would like 4-VA for supporting this work, https://4-va.org/ (Grant no. J0807483).

\section{References}

[1] W. Liu, R. Wang, R. Ding, X. Meng, and L. Yang, "On-line estimation of road profile in semi-active suspension based on unsprung mass acceleration," Mechanical Systems and Signal Processing, vol. 135, Article ID 106370, 2020.

[2] B. Zhao, T. Nagayama, and K. Xue, "Road profile estimation, and its numerical and experimental validation, by smartphone measurement of the dynamic responses of an ordinary vehicle," Journal of Sound and Vibration, vol. 457, pp. 92-117, 2019.

[3] K. Hall and C. Muñoz, "Estimation of present serviceability index from international roughness index," Transportation Research Record: Journal of the Transportation Research Board, vol. 1655, no. 1, pp. 93-99, 1999.

[4] T. Hanson, C. Cameron, and E. Hildebrand, "Evaluation of low-cost consumer-level mobile phone technology for measuring international roughness index (IRI) values," Canadian Journal of Civil Engineering, vol. 41, no. 9, pp. 819-827, 2014.

[5] J. J. Hajek, T. J. Kazmierowski, and G. Musgrove, "Switching to international roughness index," Transportation Research Record: Journal of the Transportation Research Board, vol. 1643, no. 1, pp. 116-124, 1998.

[6] S. Islam, W. G. Buttlar, R. G. Aldunate, and W. R. Vavrik, "Measurement of pavement roughness using android-based smartphone application," Transportation Research Record: Journal of the Transportation Research Board, vol. 2457, no. 1, pp. 30-38, 2014.

[7] W. Aleadelat and K. Ksaibati, "Estimation of pavement serviceability index through android-based smartphone application for local roads," Transportation Research Record: Journal of the Transportation Research Board, vol. 2639, no. 1, pp. 129-135, 2017.

[8] S. Terzi, "Modeling the pavement serviceability ratio of flexible highway pavements by artificial neural networks," Construction and Building Materials, vol. 21, no. 3, pp. 590593, 2007.

[9] L. Fuentes, R. Camargo, J. Arellana, C. Velosa, and G. Martinez, "Modelling pavement serviceability of urban roads using deterministic and probabilistic approaches,"
International Journal of Pavement Engineering, vol. 22, no. 1, pp. 77-86, 2019.

[10] H. Maeda, Y. Sekimoto, T. Seto, T. Kashiyama, and H. Omata, "Road damage detection and classification using deep neural networks with smartphone images," Computer-Aided Civil and Infrastructure Engineering, vol. 33, no. 12, pp. 1127-1141, 2018.

[11] F. E. Gunawan, Yanfi, and B. Soewito, "A vibratory-based method for road damage classification," in Proceedings of the 2015 International Seminar on Intelligent Technology and Its Applications (ISITIA), pp. 1-4, Surabaya, Indonesia, May 2015.

[12] Y. Kobana, J. Takahashi, Y. Tobe, and G. Lopez, "Accurate road damage classification based on real signal mother wavelet of acceleration signal," in Proceedings of the 2015 IEEE/SICE International Symposium on System Integration (SII), pp. 900-905, Nagoya, Japan, December 2015.

[13] H. Y. Kim, Y. F. Shen, and J. H. Ahn, "Development of a surface roughness measurement system using reflected laser beam," Journal of Materials Processing Technology, vol. 130131, no. 131, pp. 662-667, 2002.

[14] K. Xue, T. Nagayama, and B. Zhao, "Road profile estimation and half-car model identification through the automated processing of smartphone data," Mechanical Systems and Signal Processing, vol. 142, Article ID 106722, 2020.

[15] T.-Y. Chuang, N.-H. Perng, and J.-Y. Han, "Pavement performance monitoring and anomaly recognition based on crowdsourcing spatiotemporal data," Automation in Construction, vol. 106, Article ID 102882, 2019.

[16] H.-J. Kim, H. Seok Yang, and Y.-P. Park, "Improving the vehicle performance with active suspension using roadsensing algorithm," Computers \& Structures, vol. 80, no. 1819, pp. 1569-1577, 2002.

[17] T. Mimuro and T. Maemura, "Road profile measuring system using laser displacement sensors," JSAE Review, vol. 11, no. 4, 1990.

[18] Y. Li, B. Yong, H. Wu, R. An, and H. Xu, "Road detection from airborne LiDAR point clouds adaptive for variability of intensity data," Optik, vol. 126, no. 23, pp. 4292-4298, 2015.

[19] D. Stavens and S. Thrun, "A self-supervised terrain roughness estimator for off-road autonomous driving," 2012, http:// arxiv.org/abs/1206.6872.

[20] M. Doumiati, J. Martinez, O. Sename, L. Dugard, and D. Lechner, "Road profile estimation using an adaptive YoulaKučera parametric observer: comparison to real profilers," Control Engineering Practice, vol. 61, pp. 270-278, 2017.

[21] J. C. Tudón-Martínez, S. Fergani, O. Sename, J. J. Martinez, R. Morales-Menendez, and L. Dugard, "Adaptive road profile estimation in semiactive car suspensions," IEEE Transactions on Control Systems Technology, vol. 23, no. 6, pp. 2293-2305, 2015.

[22] N. Abulizi, A. Kawamura, K. Tomiyama, and S. Fujita, "Measuring and evaluating of road roughness conditions with a compact road profiler and ArcGIS," Journal of Traffic and Transportation Engineering (English Edition), vol. 3, no. 5, pp. $398-411,2016$.

[23] H. Imine, Y. Delanne, and N. K. M'Sirdi, "Road profile input estimation in vehicle dynamics simulation," Vehicle System Dynamics, vol. 44, no. 4, pp. 285-303, 2006.

[24] J. Lucas and A. Viano, "Systematic measurement of evenness on the road network: high output longitudinal profile analyser," Fr. Bridge Pavement Laboratory Report, vol. 101, 1979.

[25] N. Lushnikov and P. Lushnikov, "Methods of assessment of accuracy of road surface roughness measurement with 
profilometer," Transportation Research Procedia, vol. 20, pp. 425-429, 2017.

[26] C. Chen, Z. Liu, S. Wan, J. Luan, and Q. Pei, "Traffic flow prediction based on deep learning in Internet of vehicles," IEEE Transactions on Intelligent Transportation Systems, pp. 1-14, 2020.

[27] C. Chen, B. Liu, S. Wan, P. Qiao, and Q. Pei, “An edge traffic flow detection scheme based on deep learning in an intelligent transportation system," IEEE Transactions on Intelligent Transportation Systems, vol. 22, no. 3, pp. 1840-1852, 2021.

[28] H. M. Ngwangwa, P. S. Heyns, H. G. A. Breytenbach, and P. S. Els, "Reconstruction of road defects and road roughness classification using Artificial Neural Networks simulation and vehicle dynamic responses: application to experimental data," Journal of Terramechanics, vol. 53, pp. 1-18, 2014.

[29] H. M. Ngwangwa, P. S. Heyns, F. J. J. Labuschagne, and G. K. Kululanga, "Reconstruction of road defects and road roughness classification using vehicle responses with artificial neural networks simulation," Journal of Terramechanics, vol. 47, no. 2, pp. 97-111, 2010.

[30] A. Solhmirzaei, S. Azadi, and R. Kazemi, "Road profile estimation using wavelet neural network and 7-DOF vehicle dynamic systems," Journal of Mechanical Science and Technology, vol. 26, no. 10, pp. 3029-3036, 2012.

[31] H. M. Ngwangwa and P. S. Heyns, "Application of an ANNbased methodology for road surface condition identification on mining vehicles and roads," Journal of Terramechanics, vol. 53, pp. 59-74, 2014.

[32] M. Doumiati, A. Victorino, A. Charara, and D. Lechner, "Estimation of road profile for vehicle dynamics motion: experimental validation," in Proceedings of the 2011 American Control Conference, pp. 5237-5242, San Francisco, CA, USA, June 2011.

[33] T. Heyns, P. S. Heyns, and J. P. de Villiers, "A method for realtime condition monitoring of haul roads based on Bayesian parameter estimation," Journal of Terramechanics, vol. 49, no. 2, pp. 103-113, 2012.

[34] Y. Qin, Z. Wang, C. Xiang, E. Hashemi, A. Khajepour, and Y. Huang, "Speed independent road classification strategy based on vehicle response: theory and experimental validation," Mechanical Systems and Signal Processing, vol. 117, pp. 653-666, 2019.

[35] M. Burger, M. Speckert, R. Müller, and D. Weiberle, "Modelbased identification of road profiles and road roughness indicators using vehicle measurements," in Commercial Vehicle Technology 2018, pp. 276-287, Springer, Wiesbaden, Germany, 2018.

[36] Y. Qin, C. Wei, X. Tang, N. Zhang, M. Dong, and C. Hu, "A novel nonlinear road profile classification approach for controllable suspension system: simulation and experimental validation," Mechanical Systems and Signal Processing, vol. 125, pp. 79-98, 2019.

[37] B. Peeters and G. De roeck, "Reference-based stochastic subspace identification for output-only modal analysis," Mechanical Systems and Signal Processing, vol. 13, no. 6, pp. 855-878, 1999.

[38] P. Van Overschee and B. L. De Moor, Subspace Identification for Linear Systems: Theory-Implementation-Applications, Springer Science \& Business Media, Berlin, Germany, 2012.

[39] R. Sarlo, P. A. Tarazaga, and M. E. Kasarda, "High resolution operational modal analysis on a five-story smart building under wind and human induced excitation," Engineering Structures, vol. 176, pp. 279-292, 2018.
[40] MATLAB, 9.7.0.1190202 (R2019b), Mathworks Inc., Natick, Massachusetts, USA, 2018.

[41] A. K. Chopra, Dynamics of Structures: Theory and Applications to Earthquake Engineering, Prentice Hall, Saddle River, NJ, USA, 1995.

[42] A. Moghadam, H. G. Melhem, and A. Esmaeily, "A proof-ofconcept study on a proposed ambient-vibration-based approach to extract pseudo-free-vibration response," Engineering Structures, vol. 212, Article ID 110517, 2020. 